\title{
(2) OPEN ACCESS \\ ALLY in fighting COVID-19: magnitude of albumin decline and lymphopenia (ALLY) predict progression to critical disease
}

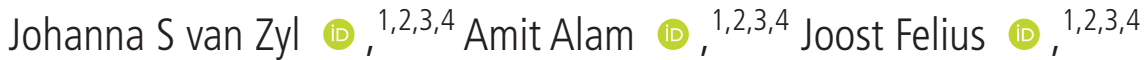 \\ Ronnie M Youssef $(1),{ }^{4}$ Dipesh Bhakta $(1),{ }^{4}$ Christina Jack, ${ }^{4}$

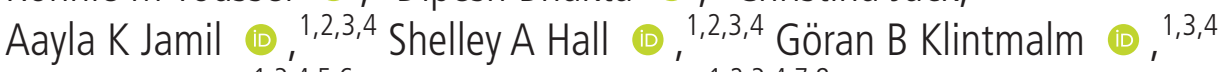 \\ Cedric W Spak, ${ }^{1,3,4,5,6}$ Robert L Gottlieb (1) 1,2,3,4,7,8
}

Additional material is published online only. To view, please visit the journal online (http://dx.doi.org/10. 1136/jim-2020-001525).

For numbered affiliations see end of article.

\section{Correspondence to} Dr Robert L Gottlieb, Center for Advanced Heart and Lung Disease, 3410 Worth St, Suite 250, Baylor University Medical Center, Dallas, TX 75246, USA; robert.gottlieb@bswhealth. org

Accepted 10 November 2020

Published Online First 11 January 2021

Check for updates

(C) American Federation for Medical Research 2021 Re-use permitted under CC BY-NC. No commercial re-use. Published by BMJ.

To cite: van Zyl JS, Alam A, Felius J,

et al. J Investig Med

2021;69:710-718.

\section{ABSTRACT}

The global severe acute respiratory syndrome coronavirus 2 (SARS-CoV-2) pandemic leading to coronavirus disease 2019 (COVID-19) is straining hospitals. Judicious resource allocation is paramount but difficult due to the unpredictable disease course. Once hospitalized, discerning which patients may progress to critical disease would be valuable for resource planning. Medical records were reviewed for consecutive hospitalized patients with COVID-19 in a large healthcare system in Texas. The main outcome was progression to critical disease within 10 days from admission. Albumin trends from admission to 7 days were analyzed using mixed-effects models, and progression to critical disease was modeled by multivariable logistic regression of laboratory results. Risk models were evaluated in an independent group. Of 153 non-critical patients, 28 (18\%) progressed to critical disease. The rate of decrease in mean baselinecorrected $(\Delta)$ albumin was $-0.08 \mathrm{~g} / \mathrm{dL} /$ day $(95 \% \mathrm{Cl}$ -0.11 to $-0.04 ; p<0.001$ ) or four times faster, in those who progressed compared with those who did not progress. A model of $\Delta$ albumin combined with lymphocyte percentage predicting progression to critical disease was validated in 60 separate patients (sensitivity, 0.70; specificity, 0.74). ALLY (delta albumin and lymphocyte percentage) is a simple tool to identify patients with COVID-19 at higher risk of disease progression when: (1) a $0.9 \mathrm{~g} / \mathrm{dL}$ or greater albumin drop from baseline within 5 days of admission or (2) baseline lymphocyte of $\leq 10 \%$ is observed. The ALLY tool identified $>70 \%$ of hospitalized cases that progressed to critical COVID-19 disease. We recommend prospectively tracking albumin. This is a globally applicable tool for all healthcare systems.

\section{INTRODUCTION}

Coronavirus disease 2019 (COVID-19) caused by the novel severe acute respiratory syndrome coronavirus 2 (SARS-CoV-2) has accumulated 44.6 million confirmed cases worldwide in a period of 10-11 months with more than $1,175,000$ deaths $(2.6 \%)$ in 219 countries as of October 30, 2020. ${ }^{1}$ On March 11, 2020, the

\section{Significance of this study}

What is already known about this subject?

- Severe acute respiratory syndrome coronavirus 2 (SARS-CoV-2) is a widespread global pandemic that has rapidly accumulated more than 10 million cases as of July 2020.

- Disease presentation is broad with critical disease characterized by acute respiratory distress syndrome and multiple organ failure.

- Demographic factors, comorbidities and inflammatory markers such as albumin, $C$ reactive protein and lymphocytes are associated with disease severity and mortality.

What are the new findings?

- In this study, we confirm that albumin level at baseline is associated with disease severity.

- Furthermore, change in albumin from baseline declines four times faster in patients who progress to critical disease state (marked by shock and the need for invasive mechanical ventilation).

- Change in albumin within 5 days from admission, alongside baseline lymphopenia, is predictive of progression to critical disease state.

How might these results change the focus of research or clinical practice?

- A dynamic fall in albumin in association with lymphopenia is a simple cost-effective assessment for healthcare systems to identify patients with coronavirus disease 2019 (COVID-19) who are at higher risk of critical disease progression towards shock and the need for mechanical ventilation. It is actionable, cost-effective, and globally applicable. We recommend albumin be tracked in trials of interventions of therapeutics for COVID-19 disease.

WHO declared this global coronavirus outbreak a pandemic. ${ }^{2}$

Patients afflicted with COVID-19 pneumonia present with a broad spectrum of clinical disease 
severity, and those who develop severe or critical disease may progress precipitously. Indeed, a substantial proportion of patients exhibit a rapid clinical deterioration to acute respiratory distress syndrome, ${ }^{3-6}$ severe pneumonia, ${ }^{5-7}$ renal failure, ${ }^{8-10}$ viral hepatitis,${ }^{11}$ and/or multiorgan failure. ${ }^{12}$

Although reports from China and Italy have suggested that demographic factors (age and gender) ${ }^{13-15}$ and comorbidities (diabetes, hypertension, coronary disease, dyspnea, tobacco smoking) ${ }^{15} 16$ are associated with COVID-19, the clinical utility of these factors is clearly imperfect as none of those risk factors are necessary nor sufficient for COVID19. While our understanding remains nascent, disease severity may be associated with markers including elevated levels of neutrophils, aspartate aminotransferase (AST), lactic acid dehydrogenase, and $\mathrm{C}$ reactive protein (CRP) and decreased levels of platelets and albumin. ${ }^{17-20}$ Furthermore, albumin levels, potassium, and lymphocytes appear to be responding in conjunction with clinical improvements post-treatment. ${ }^{719}$

While low albumin levels have been described in relation to COVID-19 disease severity, and potentially also its recovery, we noted early in our experience a substantial, time-dependent drop in albumin levels in some patients. Hence, we sought to explore the potential role of the timedependent decrease in albumin as a predictor of disease progression. We have termed this risk factor "delta albumin velocity."

\section{METHODS}

The data used for the analyses and modeling presented herein were derived from electronic medical records review approved by the Institutional Review Board of Baylor Scott and White Health (BSWH) Research Institute (IRB \#020-145) and represent a total of 12 hospitals within the BSWH system. Given the retrospective nature of this research, no informed consent was obtained from the patients. Patients were included for analysis if they were age 18 years or older, hospitalized for COVID-19 between March 13 and April 7, 2020, and followed for at least 10 days postadmission. For the purpose of this analysis, to ensure an unambiguously SARS-CoV-2 positive cohort, inclusion required a result of "detected," "positive," or similar result at least once during their illness using a reverse-transcriptase PCR (RT-PCR) assay. "Presumptive positive" and indeterminate results were insufficient for inclusion. Antigen tests were not used for inclusion. A case report of an adult patient admitted with confirmed COVID-19 disease was included to illustrate the concept of delta albumin.

Severity of COVID-19 was categorized as serious if respiratory rate was $\geq 30 / \mathrm{min}$; and/or pulse oximeter oxygen saturation $\left(\mathrm{SpO}_{2}\right)$ was $\leq 94 \%$ on ambient air; and/or the ratio of partial pressure of arterial oxygen to fraction of inspired oxygen $\left(\mathrm{PaO}_{2} / \mathrm{FiO}_{2}\right)$ was $\leq 300 \mathrm{~mm} \mathrm{Hg}$. Severity was categorized as critical if respiratory failure occurred and patient received mechanical ventilation; and/or patient was in shock defined as diagnosed by the attending physician or need of inotropic support. Hospitalized patients not meeting criteria for serious or critical disease state were categorized as moderate. Patients were identified via electronic medical record query from laboratory records detecting SARS-CoV-2 RNA merged with inpatient admission records.

\section{Data analysis}

For each subject, the following variables of interest were reviewed by chart: demographics (age, gender, ethnicity, race, body mass index), medical history and comorbidities (including asthma, coronary artery disease, history of cancer or current patient with cancer, chronic obstructive pulmonary disease, diabetes mellitus, hypertension, history of renal disease, and current or former smoker), clinical presentation at admission (including anorexia, diarrhea, chest pain, cough, fever, headache, myalgia, skin rash, shortness of breath, vomiting and nausea, respiratory rate, pulse oximeter oxygen saturation, ratio of partial pressure of arterial oxygen to fraction of inspired oxygen, shock, organ failure), laboratory assessments (SARS-CoV-2 PCR and other COVID-19 detection tests, specimen source, complete blood count, blood chemistry), and hospital and intensive care unit (ICU) admission and discharge details. To facilitate modeling of the effects of comorbidities and clinical variables, a comorbidity score was calculated as the number of comorbidities present at admission out of asthma, coronary artery disease, chronic obstructive pulmonary disease, diabetes mellitus, hypertension, cancer, history of renal disease, and smoking. Similarly, a clinical score was calculated as the number of clinical symptoms present at admission out of the presence of anorexia, chest pain, cough, diarrhea, fever, headache, myalgia, skin rash, shortness of breath, and vomiting or nausea.

Multiple organ failure was defined as two or more organs failing acutely or chronically as reported in the chart for heart, liver, kidney or respiratory failure (or acute injury) during the index admission or any subsequent readmission within 30 days. Heart failure was assessed as an ejection fraction $<35 \%$, a cardiac index $<2$, and/or the need for inotropic support including epinephrine, norepinephrine, dobutamine, dopamine, phenylephrine, vasopressin, midodrine, and isoproterenol to maintain adequate cardiac support. Hepatic injury or hepatic failure was defined as AST or alanine aminotransferase $>5$ times the upper limit of normal. Renal failure was defined as stage-5 kidney disease with a glomerular filtration rate $<15$ or need for dialysis. Respiratory failure was assessed as the initiation of mechanical ventilation or extracorporeal life membrane oxygenation.

Patients were categorized by disease severity on admission as moderate, serious or critical, and further assessed for progression to critical disease status within 10 days from admission. Baseline demographics and clinical and laboratory characteristics were reported as median (quartiles) or absolute counts (\%) by disease severity and compared using Kruskal-Wallis or $\chi^{2}$ (Fisher's exact) tests. In order to arrive at separate derivation and test sets for predictive modeling, consecutive patients admitted non-critically ill between March 13 and April 1, 2020 were assigned to the derivation set while patients admitted on April 1, 2020 and after formed the test set. For patients in the derivation set, baseline factors were compared between outcome groups defined by progression to critical disease status by 10 days postadmission using Wilcoxon-rank-sum or $\chi^{2}$ (Fisher's exact) tests. Significant factors $(\mathrm{p}<0.05)$ associated with disease progression (to critically ill) were considered in univariate and bivariate logistic regression models to develop a risk assessment model. The model with the highest area under 


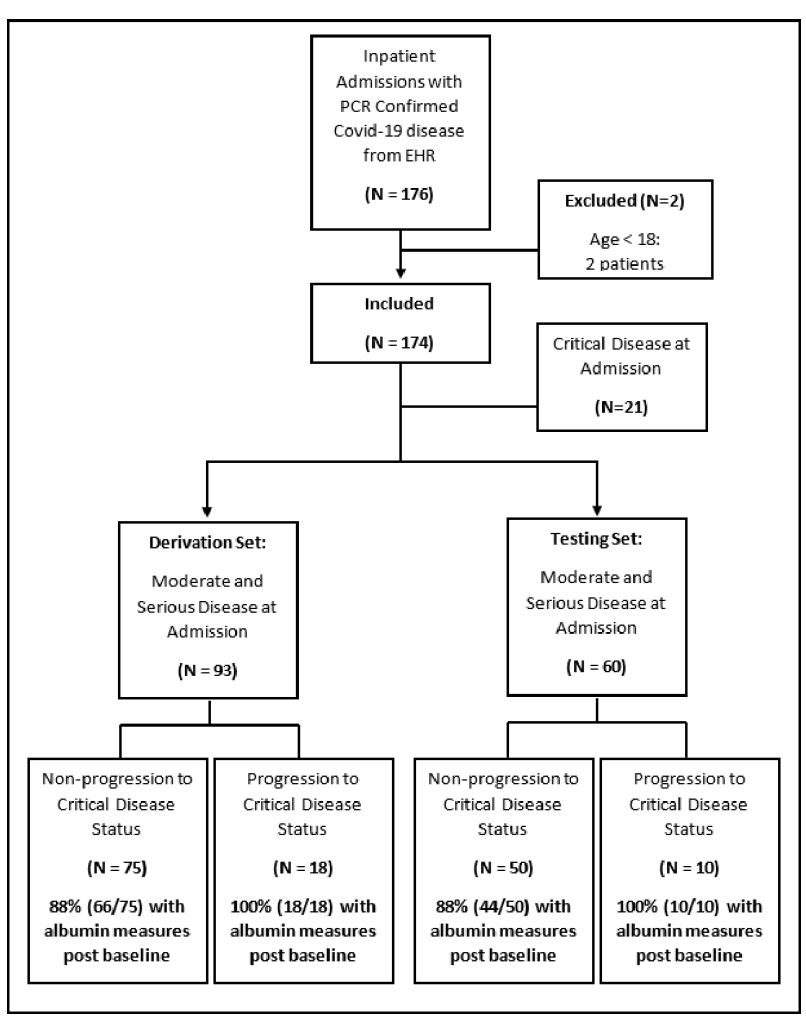

Figure 1 Subject flow diagram for the derivation and test sets.

the receiver-operator characteristic curve (AUC), and with sensitivity and specificity $\geq 50 \%$ was selected. Associations of predictor variables with disease progression were reported as ORs with 95\% Wald-confidence intervals. The predictive capacity of the model was reported in terms of sensitivity, specificity, and the AUC as determined in the independent test set derived from patients with COVID-19 admitted on April 1, 2020 or later (who met the same inclusion criteria as patients in the derivation set). DeLong's method was used to report a $95 \%$ CI for the AUC. Patients with missing values in the test set were excluded for the main predictive performance metrics. The effect of missing data on the predictive metrics was evaluated by imputing missing values with the median from the training set. Analyses were performed using R (V.3.6.1).

Differences in the rate of change in albumin within 7 days postadmission between patients who progressed to critical disease status and those who did not were modeled using a mixed-effects model. Population-averaged and subject-specific linear models were considered with compound symmetric, Toeplitz, first-order autoregressive, and Markov covariance structures characterizing within-subject variability and an unstructured covariance structure characterizing betweensubject variability in change from baseline levels of albumin. The best model with the lowest Akaike Information Criterion was selected. These analyses were performed in SAS (V.9.4) using Proc Mixed.

\section{Sample size and number of predictor variables}

A maximum number of two predictors were allowed in the logistic regression models to reduce overfitting and optimizing predictive performance ${ }^{21}$ At an expected 20\% event rate, the sample size required to ensure a margin of error $\leq 0.10$ in the overall predicted risk was 62 patients. Furthermore, the number of predictor variables were limited to two to ensure a shrinkage factor $\geq 0.9$ assuming the apparent Cox-Snell $\mathrm{R}^{2}=0.2$.

\section{RESULTS}

A total of 174 patients with COVID-19 met the eligibility criteria. Of these, 103 patients were admitted before April 1,2020 , including 10 patients critically ill on admission and 93 that entered the hospital non-critical; the 93 patients admitted non-critically ill formed the derivation set. They had a median age of 57.4 years (Q1, Q3: 48.1, 67.2), and $61(59 \%)$ were male. The flow diagram in figure 1 shows the numbers of patients included in each group for the analyses. Table 1 presents their demographics and baseline laboratory test results, and online supplemental table 1) reports on additional clinical presentation features at admission. Disease severity category at hospital admission was significantly associated with gender, anorexia, baseline levels of albumin, CRP, lymphocytes, and neutrophils, with more severe disease corresponding to lower levels of albumin and lymphocytes and higher levels of neutrophils and CRP. Almost half of the patients required advanced care with $48 \%(n=49 / 103)$ admitted to the ICU. Severe clinical outcomes such as shock ( $n=10,100 \%$ of critical patients), multi-organ failure ( $n=7 ; 70 \%$ of critical patients), and inpatient mortality $(n=5,50 \%$ of critical patients) occurred frequently in patients admitted with critical disease.

\section{Risk of progression to critical disease status by 10 days post admission}

Of the 93 patients in the derivation set admitted in moderate or serious condition, 18 (19\%) progressed to critical disease within 10 days (median (Q1, Q3): $4(2,6)$ days) postadmission. Of these, six patients expired during admission. Progression to critical disease state within 10 days was significantly associated with a clinical presentation of anorexia at admission $(p=0.04)$, higher baseline levels of neutrophils $(p=0.006)$, CRP $(p<0.001)$, and ferritin $(p=0.03)$, a larger drop in albumin $(p=0.001)$, and with lower baseline levels of oxygen saturation $(p=0.001)$, lymphocytes $(\mathrm{p}=0.01)$ and total bilirubin $(\mathrm{p}=0.04$; table 2$)$. Multivariable logistic regression modeling resulted in a two-variable model (baseline lymphocytes, $\mathrm{p}=0.01$; and albumin baseline change to nadir, $\mathrm{p}=0.002)$ showing largest AUC $(0.81,95 \%$ CI 0.70 to 0.92 ; figure $2 \mathrm{~A}$ ) with model estimates reported in table 3 . All other variables constant, a $0.1 \mathrm{~g} / \mathrm{dL}$ decrease in albumin from baseline was associated on average with a $16 \%$ increase in risk of progression to critical disease (OR, 0.84 ; $95 \%$ CI 0.59 to 0.88 ; figure $2 \mathrm{~B}$ ); similarly, each $1 \%$ decrease in lymphocytes was associated with a $9 \%$ increase in risk on average (OR, 0.91; 95\% CI 0.83 to 0.97 ; figure $2 \mathrm{~B}$ ). This risk model features a sensitivity of 0.78 and specificity of 0.71 at the risk score cut-off equal to the event rate of 0.18 in the derivation set.

\section{Validation of the lymphocyte and change in albumin risk assessment model}

The number of patients with COVID-19 meeting eligibility criteria who were admitted on April 1, 2020 or later was 71. Of these patients, 11 (15\%) presented with critical disease at admission and were excluded, with the remaining 60 patients presenting with moderate or serious disease 
Table 1 Baseline results

\begin{tabular}{|c|c|c|c|c|}
\hline \multirow[b]{3}{*}{ Variable } & \multicolumn{3}{|c|}{ Disease severity on admission } & \multirow[b]{3}{*}{$P$ value } \\
\hline & Moderate & Serious & Critical & \\
\hline & $(n=59)$ & $(n=34)$ & $(n=10)$ & \\
\hline \multicolumn{5}{|l|}{ Demographics } \\
\hline Age (years) & 58.3 (50.0 to 65.1$)$ & 52.5 (48.9 to 70.5$)$ & $48.8(40.3$ to 70.3$)$ & 0.69 \\
\hline BMI $\left(\mathrm{kg} \mathrm{m}^{-2}\right)$ & 30.7 (27.3 to 35.4$)$ & $31.0(24.7$ to 36.5$)$ & $35.0(29.5$ to 38.6$)$ & 0.52 \\
\hline Ethnicity, Hispanic or Latino & $13(22.0 \%)$ & $9(26.5 \%)$ & $4(40 \%)$ & 0.47 \\
\hline Male, gender & $30(50.9 \%)$ & $26(76.5 \%)$ & $5(50 \%)$ & 0.04 \\
\hline Race & & & & 0.74 \\
\hline Black or African American & $22(37.3 \%)$ & $10(29.4 \%)$ & $3(30 \%)$ & \\
\hline White or Caucasian & $30(50.9 \%)$ & $22(64.7 \%)$ & $6(60 \%)$ & \\
\hline Other & $7(11.9 \%)$ & $2(5.9 \%)$ & $1(10 \%)$ & \\
\hline \multicolumn{5}{|l|}{ Clinical presentation } \\
\hline Blood pressure, systolic (mm Hg) & 131 (116 to 148$)$ & 129 (119 to 147$)$ & 128 (114 to 132$)$ & 0.74 \\
\hline Blood pressure, diastolic (mm Hg) & 75 (69 to 86$)$ & 78 (69 to 88$)$ & 65 (56 to 69$)$ & 0.04 \\
\hline Clinical Score & $3(3$ to 5$)$ & $4(3$ to 5$)$ & $3(2$ to 4$)$ & 0.61 \\
\hline Respiratory rate & 20 (18 to 20$)$ & 20 (18 to 26$)$ & 23 (19 to 25$)$ & 0.07 \\
\hline $\mathrm{SpO}_{2}(\%)$ & 97 (95 to 98 ) & 93 (90 to 94$)$ & 89 (87 to 94$)$ & $<0.001$ \\
\hline \multicolumn{5}{|l|}{ Comorbidities } \\
\hline Comorbidity Score & 1 (1 to 3$)$ & 2 (1 to 2 ) & 2 (1 to 2 ) & 0.96 \\
\hline Asthma & $8(13.6 \%)$ & $2(5.9 \%)$ & $0(0 \%)$ & 0.36 \\
\hline$C A D$ & $5(8.5 \%)$ & $3(8.8 \%)$ & $2(20 \%)$ & 0.47 \\
\hline Cancer & $8(13.6 \%)$ & $2(5.9 \%)$ & $0(0 \%)$ & 0.36 \\
\hline COPD & $6(10.2 \%)$ & $1(2.9 \%)$ & $0(0 \%)$ & 0.41 \\
\hline Diabetes & $19(32.2 \%)$ & $12(35.3 \%)$ & $3(30 \%)$ & 0.95 \\
\hline Hypertension & $36(61.0 \%)$ & $17(50.0 \%)$ & $6(60 \%)$ & 0.61 \\
\hline Renal disease & $9(15.3 \%)$ & $9(26.5 \%)$ & $0(0 \%)$ & 0.14 \\
\hline Smoker & $15(25.4 \%)$ & $13(38.2 \%)$ & $4(40.0 \%)$ & 0.36 \\
\hline \multicolumn{5}{|l|}{ Laboratory features } \\
\hline Albumin (on admission) (g/dL) & 3.4 (3.2 to 3.9$)$ & 3.6 (3.0 to 3.9$)$ & $2.8(2.3$ to 3.3$)$ & 0.02 \\
\hline $\begin{array}{l}\text { Albumin change (baseline to nadir) } \\
(\mathrm{g} / \mathrm{dL})\end{array}$ & $-0.5(-0.7$ to -0.4$)$ & $-0.6(-1.0$ to -0.4$)$ & $-0.7(-1.0$ to -0.4$)$ & 0.18 \\
\hline Alkaline phosphatase (U/L) & 81 (61 to 103$)$ & 78 (55 to 96$)$ & 70 (54 to 85$)$ & 0.66 \\
\hline $\operatorname{ALT}(\mathrm{U} / \mathrm{L})$ & 32 (23 to 59$)$ & 33 (27 to 48$)$ & 44 (37 to 63$)$ & 0.79 \\
\hline AST (U/L) & 39 (23 to 58$)$ & 41 (29 to 55$)$ & 60 (41 to 134$)$ & 0.23 \\
\hline BNP $(p g / m L)$ & 51 (18 to 118$)$ & 48 (22 to 124$)$ & 82 (48 to 129 ) & 0.61 \\
\hline Creatinine (mg/dL) & $1.0(0.5$ to 3.0$)$ & 1.5 (1.0 to 2.0$)$ & 1.5 (1.0 to 2.0$)$ & 0.96 \\
\hline $\mathrm{C}$ reactive protein (mg/dL) & $5.9(2.5$ to 10.1$)$ & 11.1 (4.0 to 16.2 ) & 16.2 (6.8 to 17.2 ) & 0.03 \\
\hline Ferritin $(\mathrm{ng} / \mathrm{mL})$ & 360 (166 to 707$)$ & 531 (302 to 1183$)$ & 761 (210 to 1682$)$ & 0.27 \\
\hline Lymphocytes (\%) & 20 (13 to 29$)$ & $12(9$ to 19$)$ & 12 (9 to 18$)$ & 0.006 \\
\hline Neutrophils (\%) & 69 (61 to 79$)$ & 74 (70 to 85 ) & 80 (72 to 84$)$ & 0.007 \\
\hline \multicolumn{5}{|l|}{ Admission details } \\
\hline DNAR status & $3(5.1 \%)$ & $2(5.9 \%)$ & $1(10.0 \%)$ & 0.63 \\
\hline ICU admission & $20(33.9 \%)$ & $19(55.9 \%)$ & $10(100 \%)$ & $<0.001$ \\
\hline \multicolumn{5}{|l|}{ Critical outcomes } \\
\hline Invasive mechanical ventilation & $4(6.8 \%)$ & $14(41.2 \%)$ & $6(60.0 \%)$ & $<0.001$ \\
\hline Multiorgan failure & $5(8.5 \%)$ & $13(38.2 \%)$ & $7(70 \%)$ & $<0.001$ \\
\hline Heart failure & $3(5.1 \%)$ & $9(26.5 \%)$ & $6(60 \%)$ & $<0.001$ \\
\hline Hepatic failure & $5(8.5 \%)$ & $3(8.8 \%)$ & $1(10 \%)$ & 1.00 \\
\hline Kidney failure & $3(5.1 \%)$ & $7(20.6 \%)$ & $3(30.0 \%)$ & 0.01 \\
\hline Respiratory failure & $7(11.9 \%)$ & $15(44.1 \%)$ & $7(70 \%)$ & $<0.001$ \\
\hline Shock & $4(7.0 \%)$ & $9(25.0 \%)$ & $10(100 \%)$ & $<0.001$ \\
\hline Mortality, inpatient & $4(6.8 \%)$ & $7(20.6 \%)$ & $5(50 \%)$ & 0.003 \\
\hline
\end{tabular}

ALT, alanine aminotransferase; AST, aspartate aminotransferase; BMI, body mass index; BNP, B-type natriuretic peptide; CAD, coronary artery disease; COPD, chronic obstructive pulmonary disease; DNAR, do not attempt resuscitation; ICU, intensive care unit; NAA, nucleic acid amplification test; $\mathrm{SpO}_{2}$, pulse oximeter oxygen saturation. 
Table 2 Comparison of demographic, clinical, and laboratory factors by progression of disease

\begin{tabular}{|c|c|c|c|}
\hline \multirow[b]{2}{*}{ Variable } & \multicolumn{2}{|c|}{ Disease progression } & \multirow[b]{2}{*}{$P$ value } \\
\hline & $\begin{array}{l}\text { Remained non- } \\
\text { critical }(n=75)\end{array}$ & $\begin{array}{l}\text { Progressed to critical } \\
(n=18)\end{array}$ & \\
\hline \multicolumn{4}{|l|}{ Demographics } \\
\hline Age (years) & 57 (49 to 65$)$ & 60 (52 to 69$)$ & 0.48 \\
\hline BMI $\left(\mathrm{kg} \mathrm{m}^{-2}\right)$ & 30.9 (26.9 to 35.9$)$ & 30.9 (24.6 to 35.5$)$ & 0.67 \\
\hline $\begin{array}{l}\text { Ethnicity, Hispanic or } \\
\text { Latino }\end{array}$ & $17(22.7 \%)$ & $5(27.8 \%)$ & 0.76 \\
\hline Male gender & $42(56.0 \%)$ & $14(77.8 \%)$ & 0.15 \\
\hline Race & & & 0.39 \\
\hline $\begin{array}{l}\text { Black or African } \\
\text { American }\end{array}$ & $28(37.3 \%)$ & $4(22.2 \%)$ & \\
\hline White or Caucasian & $39(52.0 \%)$ & $13(72.2 \%)$ & \\
\hline Other & $8(10.7 \%)$ & $1(5.6 \%)$ & \\
\hline \multicolumn{4}{|l|}{$\begin{array}{l}\text { Admission clinical } \\
\text { presentation }\end{array}$} \\
\hline $\begin{array}{l}\text { Blood pressure, systolic } \\
(\mathrm{mm} \mathrm{Hg})\end{array}$ & 129 (116 to 144$)$ & 142 (123 to 155$)$ & 0.27 \\
\hline $\begin{array}{l}\text { Blood pressure, diastolic } \\
(\mathrm{mm} \mathrm{Hg})\end{array}$ & 76 (68 to 87 ) & 81 (73 to 85$)$ & 0.51 \\
\hline Clinical Score & 3 (3 to 5$)$ & 4 (3 to 4$)$ & 0.60 \\
\hline Anorexia & $11(14.7 \%)$ & $7(38.9 \%)$ & 0.04 \\
\hline Chest pain & $13(17.3 \%)$ & $0(0 \%)$ & 0.07 \\
\hline Cough & $58(77.3 \%)$ & $16(88.9 \%)$ & 0.35 \\
\hline Diarrhea & $20(26.7 \%)$ & $3(16.7 \%)$ & 0.55 \\
\hline Fever & $61(81.3 \%)$ & $14(77.8 \%)$ & 0.74 \\
\hline Headache & $12(16.0 \%)$ & $3(16.7 \%)$ & 1.00 \\
\hline Myalgia & $22(29.3 \%)$ & $7(38.9 \%)$ & 0.62 \\
\hline Rash & $0(0 \%)$ & $1(5.6 \%)$ & 0.19 \\
\hline Shortness of breath & $53(70.7 \%)$ & $12(66.7 \%)$ & 0.96 \\
\hline Nausea/Vomiting & $18(24 \%)$ & $5(27.8 \%)$ & 0.77 \\
\hline Respiratory rate & 19 (18 to 22$)$ & 20 (18 to 22$)$ & 0.36 \\
\hline $\mathrm{SpO}_{2}(\%)$ & 96 (95 to 97) & 94 (90 to 96) & 0.001 \\
\hline \multicolumn{4}{|l|}{ Comorbidities } \\
\hline Comorbidity Score & 1 (1 to 3 ) & 2 (1 to 4 ) & 0.36 \\
\hline Asthma & $10(13.3 \%)$ & $0(0 \%)$ & 0.20 \\
\hline CAD & $6(8.0 \%)$ & $2(11.1 \%)$ & 0.65 \\
\hline Cancer & $9(12.0 \%)$ & $1(5.6 \%)$ & 0.68 \\
\hline COPD & $5(6.7 \%)$ & $2(11.1 \%)$ & 0.62 \\
\hline Diabetes & $21(28.0 \%)$ & $10(55.6 \%)$ & 0.05 \\
\hline Hypertension & $42(56.0 \%)$ & $11(61.1 \%)$ & 0.90 \\
\hline Renal disease & $14(18.7 \%)$ & $4(22.2 \%)$ & 0.74 \\
\hline Smoker & $21(28.0 \%)$ & $7(38.9 \%)$ & 0.54 \\
\hline $\begin{array}{l}\text { Disease severity on } \\
\text { admission }\end{array}$ & & & $<0.001$ \\
\hline Moderate & $55(73.3 \%)$ & $4(22.2 \%)$ & \\
\hline Serious & $20(26.7 \%)$ & $14(77.8 \%)$ & \\
\hline \multicolumn{4}{|l|}{ Laboratory results } \\
\hline $\begin{array}{l}\text { Albumin (on admission) } \\
\text { (g/dL) }\end{array}$ & 3.5 (3.1 to 3.9$)$ & 3.4 (3.0 to 3.9$)$ & 0.72 \\
\hline $\begin{array}{l}\text { Albumin change } \\
\text { (baseline to nadir) } \\
\text { (g/dL) }\end{array}$ & $-0.5(-0.7$ to -0.3$)$ & $-0.9(-1.1$ to -0.5$)$ & 0.001 \\
\hline $\begin{array}{l}\text { Alkaline phosphatase } \\
\text { (U/L) }\end{array}$ & 81 (60 to 99) & 73 (57 to 106) & 0.91 \\
\hline ALT (U/L) & 32 (24 to 52 ) & 39 (27 to 80$)$ & 0.32 \\
\hline AST (U/L) & 39 (25 to 56$)$ & 42 (31 to 124$)$ & 0.15 \\
\hline BNP (pg/mL) & 53 (20 to 123$)$ & 38 (21 to 76$)$ & 0.46 \\
\hline Creatinine (mg/dL) & $1.0(0.8$ to 1.3$)$ & 1.1 (1.0 to 1.8$)$ & 0.28 \\
\hline $\begin{array}{l}\text { C reactive protein } \\
(\mathrm{mg} / \mathrm{dL})\end{array}$ & 4.7 (2.4 to 9.0$)$ & 16.2 (12.6 to 21.4$)$ & $<0.001$ \\
\hline Ferritin (ng/mL) & 391 (170 to 576$)$ & 970 (369 to 1741$)$ & 0.03 \\
\hline
\end{tabular}

Continued
Table 2 Continued

\begin{tabular}{|c|c|c|c|}
\hline \multirow[b]{2}{*}{ Variable } & \multicolumn{2}{|c|}{ Disease progression } & \multirow[b]{2}{*}{$P$ value } \\
\hline & $\begin{array}{l}\text { Remained non- } \\
\text { critical }(n=75)\end{array}$ & $\begin{array}{l}\text { Progressed to critical } \\
(n=18)\end{array}$ & \\
\hline Lymphocytes (\%) & 19 (12 to 27$)$ & 11 (9 to 19$)$ & 0.01 \\
\hline Neutrophils (\%) & 70 (62 to 79$)$ & 79 (71 to 87$)$ & 0.006 \\
\hline Total bilirubin (mg/dL) & 0.5 (0.4 to 0.7$)$ & 0.6 (0.6 to 0.9$)$ & 0.04 \\
\hline Troponin-I (ng/mL) & $0.02(0.02$ to 0.03$)$ & 0.02 (0.01 to 0.04$)$ & 0.90 \\
\hline
\end{tabular}

forming the test set for model validation. Of these, 10 (17\%) progressed to critical status within 10 days (median (Q1, Q3): $3(2,6)$ days). Baseline characteristics and critical outcomes did not differ significantly compared with the derivation cohort with the exception of older patients admitted during the validation period (median age (Q1, Q3), 71.2 years $(51.6,86.5)$; online supplemental table 2 ). The risk assessment model with baseline lymphocyte (\%) and largest drop in albumin value within 5 days from admission were used to predict progression to critical status in this test set with a sensitivity of 0.70 and specificity of 0.74 (AUC $(95 \% \mathrm{CI}): 0.78(0.61$ to 0.95$))$ using a probability cut-off of 0.18 . The sensitivity was not affected by missing data, whereas the specificity lowered slightly to 0.70 when the median change to lowest level in albumin of $-0.6 \mathrm{~g} / \mathrm{dL}$ from the derivation set was used for imputation for eight non-critical patients missing a follow-up albumin lab within 5 days from admission.

\section{Sensitivity of model prediction results to timing of change in albumin}

Overall, the median time to progression to critical disease was 3 days (Q1, Q3: 2, 6). To explore whether the drop in albumin was predictive (eg, manifest before criteria for critical disease), we assessed the maximal change in albumin from baseline within 5 days and compared it to the maximal change in albumin within 5 days manifest before developing critical status. The median difference of $0 \mathrm{~g} / \mathrm{dL}$ (Q1, Q3: 0 , $-0.2 ; \mathrm{p}=0.51)$ was not significantly different. Additionally, the precritical decrease in albumin from baseline remained significantly greater in patients who progressed to critical disease compared with non-progression patients $(p=0.007$; online supplemental table 3). Furthermore, using precritical delta-albumin in the ALLY (delta albumin and lymphocyte percentage) model to classify patients according to risk for progression to critical disease status resulted in similar model characteristics (overall AUC (95\% CI): 0.76 (0.66 to 0.86 ); online supplemental table 4 ), thus indicating that the change in albumin could be restricted to the portion that occurred before patients progressed to critical disease, consistent with characteristics of a predictive model.

\section{Change in albumin as an independent predictor of progression to critical disease status}

In the online supplemental table 5 , we report the association of change in albumin from baseline with progression to critical disease status adjusted for anorexia, diabetes mellitus, disease severity on admission, baseline levels of oxygen saturation, CRP, ferritin, neutrophil percentage, and total 

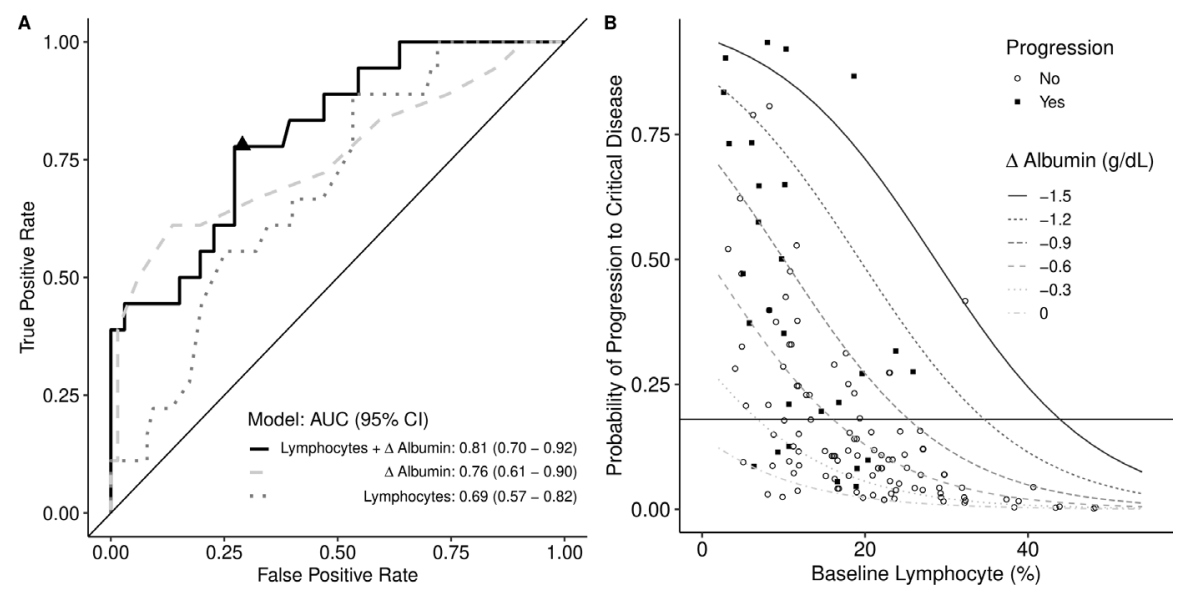

Figure 2 Risk prediction of progression to critical disease status by 10 days post admission. (A) Receiver operating curve for best multivariable logistic regression and subcomponent models. The best multivariate model consists of lymphocyte (\%) at baseline and the change in albumin from baseline to nadir. The triangle indicates the sensitivity $(0.78)$ and specificity $(0.71)$ at a probability cut-off at the event rate of 0.18 . (B) Predicted risk based on the best multivariable logistic model with baseline lymphocyte (\%) and delta albumin (change from baseline to lowest level within 5 days, g/dL). Predicted risk above 0.18 (dashed line) indicates high risk for progression to critical disease (ie, a patient with a $-1 \mathrm{~g} / \mathrm{dL}$ drop in albumin from baseline and baseline lymphocyte of $20 \%$ has a high risk probability of 0.34 ( $>0.18$ ) of progression to critical disease). AUC, area under the receiver-operator characteristic curve.

bilirubin (all $\mathrm{p}<0.05$; table 2) using two-variable models. Change in albumin remained independently associated with progression to critical disease following adjustment of each of these factors $(\mathrm{p}<0.02$ in all models; online supplemental table 5).

\section{Association of albumin decrease post admission with progression to critical disease status}

In 138 of the 153 patients admitted in moderate or serious condition, a series of follow-up laboratory measurements were acquired as part of standard of care with a median $(\mathrm{Q} 1, \mathrm{Q} 3)$ of $5(3,6)$ albumin measures per patient (figure 3A,B) for a total of 628 measurements. Mean change from baseline albumin measures decreased significantly by $-0.08(-0.11$ to -0.04$) \mathrm{g} / \mathrm{dL}$ per day in the group that progressed to critical disease compared with a stabilized trend of slower change from baseline albumin measures in the non-progression group (slope $(95 \% \mathrm{CI}):-0.02(-0.03$ to 0.01$)$ ). The rate of decrease from baseline albumin measures was $0.06 \mathrm{~g} / \mathrm{dL}(0.02,0.10 ; p=0.003$; figure $3 \mathrm{C})$ per day faster in patients who progressed to critical disease compared with non-progression patients. Moreover, the change from baseline to lowest level within 5 days was significantly larger in patients who progressed to critical disease $(p<0.001$; figure $3 D)$.

\begin{tabular}{llllll}
\hline \multicolumn{5}{l}{$\begin{array}{l}\text { Table } 3 \\
\text { development of critical disease }\end{array}$} & \multicolumn{4}{l}{ Multivariable logistic model estimates of risk for } \\
\hline Variable & Estimate & SE & OR $(95 \%$ Cl) & P value \\
\hline Intercept & -1.77 & 0.94 & $0.17(0.02$ to 0.99$)$ & 0.06 \\
\hline $\begin{array}{l}\text { Baseline lymphocyte } \\
\text { (\%) }\end{array}$ & -0.10 & 0.04 & $0.91(0.83$ to 0.97$)$ & 0.01 \\
$\begin{array}{l}\text { Total albumin change } \\
\text { (baseline to nadir) } \\
\text { (g/dL) }\end{array}$ & -3.07 & 0.98 & $0.05(0.01$ to 0.26$)$ & 0.002 \\
\hline
\end{tabular}

\section{Illustration of the risk score and albumin recovery}

A patient with a history of hyperlipidemia presented with shortness of breath and hypoxemia. The patient was subsequently admitted with serious disease at admission with a $\mathrm{SpO}_{2}$ of $83 \%$, respiratory rate of 32 breaths/min and a primary diagnosis of pneumonia due to SARS-CoV-2 confirmed by RT-PCR. Baseline lymphocyte of $14 \%$ and maximum drop in albumin of $-0.5 \mathrm{~g} / \mathrm{dL}$ within the first 5 days of admission were observed. Based on these measures, the probability of progression to critical disease within 10 days is 0.17 which falls within the lower risk category (below the threshold of 0.18). The patient did not develop shock or the need for invasive mechanical ventilation within 10 days from admission. Furthermore, albumin measures recovered to $3.2 \mathrm{~g} / \mathrm{dL}$ by day 11 at which point the patient was successfully weaned from nasal cannula oxygen support to room air (online supplemental figure S1).

\section{DISCUSSION}

We found that patients with COVID-19 who present without critical illness rapidly develop a relative hypoalbuminemia averaging $0.4 \mathrm{~g} / \mathrm{dL}$ between presentation and hospital day 1 . Subsequent to this initial drop, those patients who proceed to develop critical illness from COVID-19 pneumonia and manifest severe acute respiratory distress generally had a continued, steeper descent (mean $-0.08 \mathrm{~g} / \mathrm{dL} /$ day) of serum albumin levels compared with patients who did not progress to critical illness $(-0.02 \mathrm{~g} / \mathrm{dL} /$ day $)$. Additionally, our results confirm earlier findings in Asia and Europe that showed significant associations of disease severity (at admission) with elevated levels of neutrophils and lower levels of albumin and lymphocytes. ${ }^{17-19} 22$

Albumin is a well-known marker of risk in multiple disease states, including heart failure, ${ }^{23}{ }^{24}$ liver failure, ${ }^{25}$ other forms of sepsis, ${ }^{26}$ and frailty. ${ }^{27}{ }^{28}$ Our risk model expands on these previous observations (for this and other disease 
A

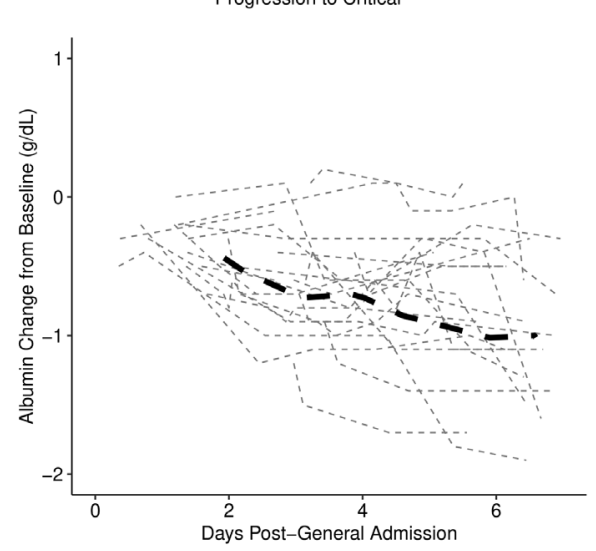

C

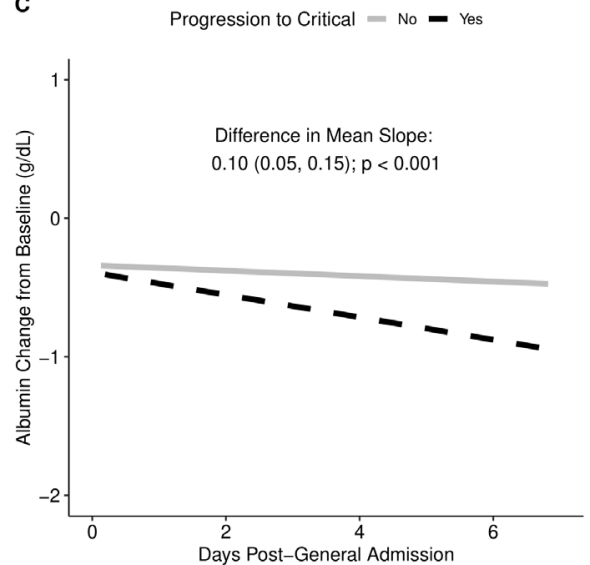

B

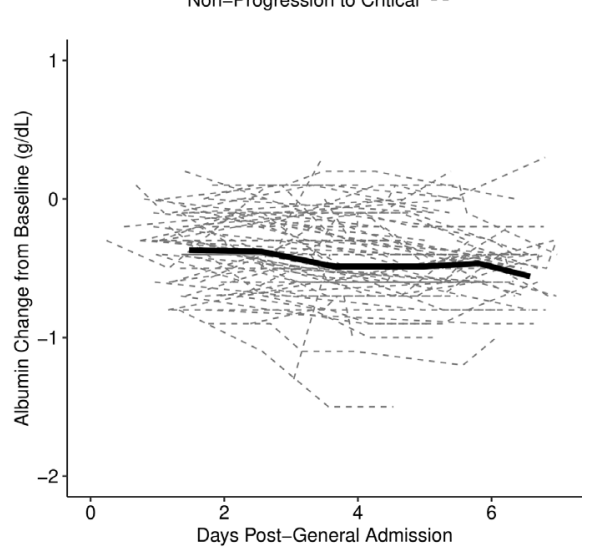

D

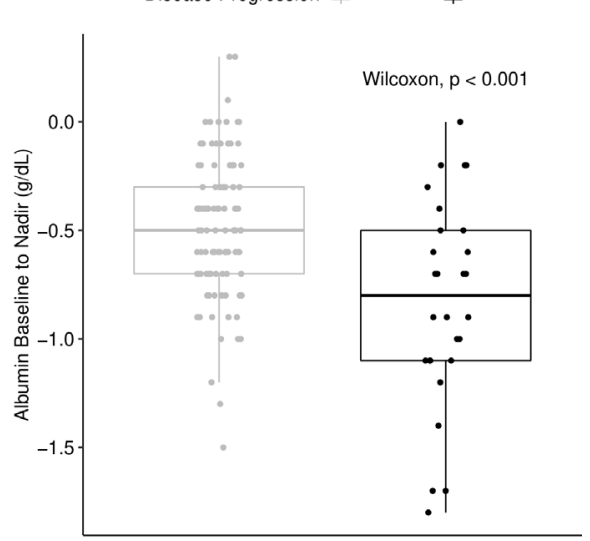

Figure 3 Association of the decrease in mean albumin from baseline within 7 days postadmission. $(A, B)$ Observed subject-specific profiles by patients who progressed to (A) critical disease and (B) those who did not with an overall mean between subsequent albumin change from baseline measures. (C) Predicted decrease from baseline and rate of change in albumin by time from admission and progression to critical disease subgroups. The change in albumin from baseline decreases by $0.06 \mathrm{~g} / \mathrm{dL}(0.02,0.10 ; \mathrm{p}=0.003)$ per day faster in the critical progression group compared with those who do not progress. In fact, the mean change from baseline in albumin does not decrease significantly from the initial change following admission in the non-progression group (slope: $-0.02(-0.03,0.01) ; p=0.01$ ) compared with a rate of change of $-0.08(-0.11$ to -0.04$) \mathrm{g} / \mathrm{dL}$ in the progression group. (D) Association of change from baseline to nadir albumin within 5 days with progression to critical disease status. The median fall in albumin is significantly larger in patients who progress to critical disease $(-0.8(-1.1,-0.5)$ vs $-0.5(-0.7,-0.3) \mathrm{g} / \mathrm{dL} ; \mathrm{p}<0.001)$.

states) in that daily changes of albumin levels are typically known to be stable or mild. In contrast, a more dynamic and precipitous fall in daily albumin levels was noted during the inflammatory response to COVID-19 pneumonia. The velocity of albumin change likely reflects overall capillary permeability ("capillary leak"), although it is also possible that the hepatic production of albumin may be impacted. The relative balance of production, clearance, and leak may be a fruitful avenue of future exploration. Although theoretically, the drop in albumin can potentially be dilutional from exogenous intravenous fluid, due to the retrospective nature of our data and the limitations from the infectious nature of the study disease, assessment of input and output and daily weights does not allow us to completely exclude this possibility. Generally, however, the management of patients with pneumonia or respiratory failure is biased towards diuresis or at least avoidance of excessive intravenous fluids. ${ }^{29}{ }^{30}$ We hypothesize that the observed albumin decrement is predominantly not dilutional from exogenous intravenous fluids. Future studies are warranted to explore the precise mechanisms for the change in albumin.

Traditional risk factors, such as oxygen saturation and clinical metrics, may be insufficient to assess the risk of decompensation in an individual admitted with COVID19. To balance patient safety and resource management, it would be expressly desirable to discern which patients can safely be discharged to convalesce at home and distinguish those from patients who are at increased risk of abrupt early or late decompensation from cytokine storm, appearing to be an unchecked inflammatory host response to viral antigen-driven stimulation. It would also be beneficial to have short-term surrogate measures for improvement and response to therapy, particularly for patients with elevated inflammatory or cytokine biomarkers. Notably, we could not correlate the magnitude and rate of albumin change with inflammatory biomarkers such as CRP or interleukin-6 (IL6) that were not consistently available over the course of admission. 
We have demonstrated a significantly faster drop in albumin in patients who progress to critical disease and retrospectively developed and validated a risk-scoring tool, which consists of lymphocyte percentage and change in albumin levels. These results thus suggest immediate, actionable findings. Albumin and lymphocyte percentage are associated with the progression of COVID-19 disease and, as such, can assist the healthcare team to discern whether a patient is at increased risk of decompensating.

Our simple set of guidelines, termed the ALLY guidelines, aims to alert the physician at the point of care if a patient is at higher risk of disease progression when: (1) a drop in albumin greater than $0.9 \mathrm{~g} / \mathrm{dL}$ from baseline occurs within the first 5 days of admission or (2) a low baseline lymphocyte of $10 \%$ or less is observed. These guidelines identified more than $70 \%$ of cases that progressed to critical disease in our set. In contrast, high baseline lymphocyte showed a protective effect with $30 \%$ or greater at admission representing a lower risk of progression to critical disease. More detailed risk assessment can be performed by applying the risk model. Second, we propose that this risk model can develop into a prospective tool to further aid discerning progression from non-critical disease to critical disease, and that it is useful as an early gauge globally, as it is readily available across different types of healthcare systems (in contrast to, for example, CRP and IL6). It remains to be seen if albumin recovery may serve as a surrogate for efficacy of a given therapeutic intervention.

An important limitation to this work includes the observational period of the study relative to the timeline of the SARS-CoV-2 pandemic. This analysis was performed at a time when steroids were discouraged by WHO guidelines. In the meantime, remdesivir has become standard of care when available for severe COVID-19, and steroid use for patients with oxygendependence has become nearly universal. Given these interval changes in standard of care, it is possible that the dynamic changes to albumin and association with outcomes may have been impacted and will require future analysis.

Furthermore, considering the dynamic changes over time in albumin levels, the timing of albumin assessment is an important component to consider in risk assessment. In patients who progressed to critical disease within 10 days of admission, the change in albumin that occurred within 5 days of admission (without censoring for when they became critically ill) was not significantly different from the change in albumin within 5 days that occurred prior to progression to critical illness. Similar predictive characteristics using precritical delta albumin suggests that delta albumin within 5 days is an appropriate surrogate for decline to critical disease state. Additionally, based on the result of a significantly slower decline in albumin levels for non-progression patients, we hypothesize that in those cases where patient discharge is being considered prior to 5 days, a qualitative assessment of magnitude of albumin decrement-to-date, even if fewer than 5 days have elapsed, may be an acceptable surrogate to assess the decision to discharge.
Finally, our model was limited to two variables to reduce overfitting with a small-to-modest study size. Despite implementing a bivariable model, the predictive metrics of ALLY performed well with a sensitivity and specificity of at least $70 \%$ in the derivation and test data. ALLY is intended to provide supplemental guidance by assessing changes in albumin and baseline lymphocyte percentage as it relates to the risk of progression to critical disease status. Other variables that were also associated with a risk for progression to critical disease status in this study and other reports included increased CRP ${ }^{17}{ }^{18}$ ferritin, ${ }^{31}$ neutrophil percentage, ${ }^{16} 17$ and severe disease status on admission. ${ }^{17}$ These variables should not be disregarded in clinical decision-making.

Although we acknowledge that this risk score will be imperfect, like all scoring systems necessarily are, we would urge colleagues to follow albumin levels periodically for changes from admission, and to reassess at least once before discharge. If an apparently stable patient is on the precipice of discharge, the overall magnitude and rate of albumin change ("delta albumin velocity") may prompt healthcare providers to carefully reconsider their decision to transition a patient to the outpatient setting, and increase the accuracy of appropriate triage decisions. Finally, we propose that the variables of interest identified in our risk model should be included as risk markers in future treatment studies for COVID-19.

\section{Author affiliations}

${ }^{1}$ Baylor Scott \& White Research Institute, Baylor Scott and White Health, Dallas, Texas, USA

${ }^{2}$ Center for Advanced Heart and Lung Disease, Baylor University Medical Center, Dallas, Texas, USA

${ }^{3}$ Baylor Annette C and Harold C Simmons Transplant Institute, Baylor Scott and White Health, Dallas, Texas, USA

${ }^{4}$ College of Medicine, Texas A\&M Health Science Center, Texas A\&M University, Dallas, Texas, USA

${ }^{5}$ Division of Infectious Disease, Baylor University Medical Center, Dallas, Texas, USA

${ }^{6}$ Texas Centers for Infectious Disease Associates, Dallas, Texas, USA

${ }^{7}$ Division of Precision Medicine, Baylor University Medical Center, Dallas, Texas, USA

${ }^{8}$ Department of Internal Medicine, TCU and UNTHSC School of Medicine, Fort Worth, Texas, USA

Contributors JSVZ, AA, RY, DB, and CJ reviewed patient charts and collected data. JSVZ and AKJ designed the data registry. AKJ was responsible for regulatory support. JSvZ analyzed the data and developed the risk model. JSVZ, JF, SAH, GBK, CWS, and RLG interpreted the results and were major contributors to writing the manuscript. JF and RLG initiated the project to validate or refute preliminary observations by RLG. All authors read and approved the final manuscript.

Funding The authors have not declared a specific grant for this research from any funding agency in the public, commercial or not-for-profit sectors.

Competing interests None declared.

Patient consent for publication Not required.

Provenance and peer review Not commissioned; externally peer reviewed.

Data availability statement Data may be obtained from a third party and are not publicly available. The datasets analyzed during the current study are protected by state and national laws within Baylor Scott and White Health. Permission from Baylor Scott and White will have to be obtained to share data.

Supplemental material This content has been supplied by the author(s). It has not been vetted by BMJ Publishing Group Limited (BMJ) and may not have been peer-reviewed. Any opinions or recommendations discussed are solely those of the author(s) and are not endorsed by BMJ. BMJ disclaims all 
liability and responsibility arising from any reliance placed on the content. Where the content includes any translated material, BMJ does not warrant the accuracy and reliability of the translations (including but not limited to local regulations, clinical guidelines, terminology, drug names and drug dosages), and is not responsible for any error and/or omissions arising from translation and adaptation or otherwise.

Open access This is an open access article distributed in accordance with the Creative Commons Attribution Non Commercial (CC BY-NC 4.0) license, which permits others to distribute, remix, adapt, build upon this work noncommercially, and license their derivative works on different terms, provided the original work is properly cited, an indication of whether changes were made, and the use is non-commercial. See: http://creativecommons.org/ licenses/by-nc/4.0/.

\section{ORCID iDs}

Johanna S van Zyl http://orcid.org/0000-0003-4205-9214

Amit Alam http://orcid.org/0000-0002-3040-3957

Joost Felius http://orcid.org/0000-0001-7200-8755

Ronnie M Youssef http://orcid.org/0000-0002-8460-5810

Dipesh Bhakta http://orcid.org/0000-0001-7512-7514

Aayla K Jamil http://orcid.org/0000-0001-9471-3383

Shelley A Hall http://orcid.org/0000-0002-5989-2934

Göran B Klintmalm http://orcid.org/0000-0003-0916-6042

Robert L Gottlieb http://orcid.org/0000-0001-8376-8709

\section{REFERENCES}

1 World Health Organization. Coronavirus disease (COVID-19) pandemic, 2020. Available: https://www.who.int/emergencies/diseases/novel-coronavirus-2019 [Accessed 30 Oct 2020].

2 World Health Organization. Virtual press conference on COVID-19, 2020. Available: https://www.who.int/docs/default-source/coronaviruse/transcripts/ who-audio-emergencies-coronavirus-press-conference-full-and-final11 mar2020.pdf

3 Goh KJ, Choong MC, Cheong EH, et al. Rapid progression to acute respiratory distress syndrome: review of current understanding of critical illness from COVID-19 infection. Ann Acad Med Singap 2020;49:1-9.

4 Li X, Ma X. Acute respiratory failure in COVID-19: is it "typical" ARDS? Crit Care 2020;24:198.

5 Rodriguez-Morales AJ, Cardona-Ospina JA, Gutiérrez-Ocampo E, et al. Clinical, laboratory and imaging features of COVID-19: a systematic review and metaanalysis. Travel Med Infect Dis 2020;34:101623.

6 Shi H, Han X, Jiang N, et al. Radiological findings from 81 patients with COVID-19 pneumonia in Wuhan, China: a descriptive study. Lancet Infect Dis 2020;20:425-34

7 Liu F, Xu A, Zhang Y, et al. Patients of COVID-19 may benefit from sustained Lopinavir-combined regimen and the increase of eosinophil may predict the outcome of COVID-19 progression. Int J Infect Dis 2020;95:183-91.

8 Yang F, Shi S, Zhu J, et al. Analysis of 92 deceased patients with COVID-19. J Med Virol 2020

9 Chen Y-S, Lin J-W, Yu H-Y, et al. Cardiopulmonary resuscitation with assisted extracorporeal life-support versus conventional cardiopulmonary resuscitation in adults with in-hospital cardiac arrest: an observational study and propensity analysis. Lancet 2008;372:554-61.

10 Al-Quteimat OM, Amer AM. The impact of the COVID-19 pandemic on cancer patients. Am J Clin Oncol 2020;43:452-5.
11 Lagana SM, De Michele S, Lee MJ, et al. COVID-19 associated hepatitis complicating recent living donor liver transplantation. Arch Pathol Lab Med 202010.5858/arpa.2020-0186-SA. [Epub ahead of print: 17 Apr 2020].

12 Chen T, Wu D, Chen H, et al. Clinical characteristics of 113 deceased patients with coronavirus disease 2019: retrospective study. BMJ 2020;368:m1091.

13 Lippi G, Mattiuzzi C, Sanchis-Gomar F, et al. Clinical and demographic characteristics of patients dying from COVID-19 in Italy versus China. J Med Virol 2020.

14 Bialek S, Boundy E, Bowen V, et al. Severe outcomes among patients with coronavirus disease 2019 (COVID-19) - United States, February 12-March 16, 2020. MMWR Morb Mortal Wkly Rep 2020;69:343-6.

15 Zhou F, Yu T, Du R, et al. Clinical course and risk factors for mortality of adult inpatients with COVID-19 in Wuhan, China: a retrospective cohort study. Lancet 2020;395:1054-62.

16 Wu C, Chen X, Cai Y, et al. Risk factors associated with acute respiratory distress syndrome and death in patients with coronavirus disease 2019 pneumonia in Wuhan, China. JAMA Intern Med 2020.

17 Mo P, Xing Y, Xiao Y, et al. Clinical characteristics of refractory COVID-19 pneumonia in Wuhan, China. Clin Infect Dis 2020. doi:10.1093/cid/ciaa270. [Epub ahead of print: 16 Mar 2020].

18 Liu W, Tao Z-W, Wang L, et al. Analysis of factors associated with disease outcomes in hospitalized patients with 2019 novel coronavirus disease. Chin Med J 2020;133:1032-8.

19 Zhu J, Ji P, Pang J, et al. Clinical characteristics of 3,062 COVID-19 patients: a meta-analysis. J Med Virol 2020.

20 Wang L. C-Reactive protein levels in the early stage of COVID-19. Médecine et Maladies Infectieuses 2020;50:332-4.

21 Riley RD, Snell KI, Ensor J, et al. Minimum sample size for developing a multivariable prediction model: PART II - binary and time-to-event outcomes. Stat Med 2019:38:1276-96.

22 Grasselli G, Zangrillo A, Zanella A, et al. Baseline characteristics and outcomes of 1591 patients infected with SARS-CoV-2 admitted to ICUs of the Lombardy region, Italy. JAMA 2020;323:1574.

23 Uthamalingam S, Kandala J, Daley M, et al. Serum albumin and mortality in acutely decompensated heart failure. Am Heart J 2010;160:1149-55.

24 Arques $S$, Ambrosi P. Human serum albumin in the clinical syndrome of heart failure. J Card Fail 2011;17:451-8.

25 Johnson PJ, Berhane S, Kagebayashi C, et al. Assessment of liver function in patients with hepatocellular carcinoma: a new evidence-based Approach-The ALBI grade. JCO 2015;33:550-8.

26 Artero A, Zaragoza R, Camarena JJ, et al. Prognostic factors of mortality in patients with community-acquired bloodstream infection with severe sepsis and septic shock. J Crit Care 2010;25:276-81.

27 Mitnitski A, Collerton J, Martin-Ruiz C, et al. Age-related frailty and its association with biological markers of ageing. BMC Med 2015;13:161.

28 Hazzard WR. Depressed albumin and high-density lipoprotein cholesterol: signposts along the final common pathway of frailty. J Am Geriatr Soc 2001:49:1253-4.

29 Alhazzani W, Møller MH, Arabi YM, et al. Surviving sepsis campaign: guidelines on the management of critically ill adults with coronavirus disease 2019 (COVID-19). Intensive Care Med 2020;46:854-87.

30 Hasanin A, Mostafa M. Evaluation of fluid responsiveness during COVID-19 pandemic: what are the remaining choices? J Anesth 2020;34:758-64.

31 Cheng L, Li H, Li L, et al. Ferritin in the coronavirus disease 2019 (COVID-19): a systematic review and meta-analysis. J Clin Lab Anal 2020;34:e23618. 